\title{
meso-四(对烷氧基苯基)卟啉镁配合物的合成、表征和性能研究
}

\author{
刘捷频 ${ }^{a, b}$ 杨德亮 ${ }^{b} \quad$ 曹 华 $b, c$ 赵鸿斌*, \\ ( ${ }^{a}$ 湘潭职业技术学院医技学院 湘潭 411103) \\ ( ${ }^{b}$ 湘潭大学化学学院 湘潭 411105)

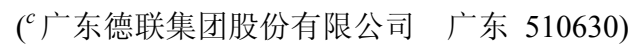

\begin{abstract}
摘要 合成了一系列 meso-四(对烷氧基苯基)卟啉镁配合物, 用 UV-Vis, ${ }^{1} \mathrm{H}$ NMR, IR, MS, 元素分析等确证了配合物的 结构, 总结了镁与卟啉类配体配合的 UV-Vis, ${ }^{1} \mathrm{H}$ NMR, IR 数据. 采用差示扫描量热法(DSC)和偏光显微镜(PM)研究了 该系列配合物的液晶性能, 发现 6 个配合物具有液晶性; 考察了烷氧基链长、配位金属离子和分子空间结构对液晶性 能的影响.
\end{abstract}

关键词 卟啉镁配合物; 合成; 表征; 液晶性

\section{Synthesis, Characterization and Properties of Metallic Magnesium Complexes of meso-Tetrakis(4-alkoxyphenyl)porphyrins}

\author{
Liu, Jiepin ${ }^{a, b} \quad$ Yang, Deliang $^{b} \quad$ Cao, Hua ${ }^{b, c} \quad$ Zhao, Hongbin ${ }^{*, b}$ \\ ( ${ }^{a}$ Medical Technology Institute, Xiangtan Vocational \& Technical College, Xiangtan 411103) \\ ( ${ }^{b}$ College of Chemistry, Xiangtan University, Xiangtan 411105) \\ ( ${ }^{c}$ Guangdong Delian Group Co., Ltd, Guangdong 510630)
}

\begin{abstract}
A series of magnesium complexes of porphyrins were synthesized. Molecular structures of all synthesized compounds were confirmed by UV-Vis, ${ }^{1} \mathrm{H}$ NMR, IR, MS techniques and elemental analysis. Characteristic spectrometric data of IR, UV-Vis, ${ }^{1} \mathrm{H}$ NMR related to their structures have been systematically analyzed. Differential scanning calorimetry (DSC) and polarizing microscope (PM) were used to probe the liquid crystal properties of the serial complexes. Six of the serial complexes were found to exhibit liquid crystal properties. The effects of alkoxy chains, metal ions of complexes and molecular space structure on liquid crystal properties have been investigated.

Keywords magnesium complexes of porphyrin; synthesis; characterization; liquid crystal property
\end{abstract}

卟啉类化合物具有特殊的 $18 \pi$ 电子共轭芳香性结 构、立体构型的复杂性、周边基团的多样性以及自组装 和分子识别 ${ }^{[1]}$ 等特性，以其为基础进行功能性分子设计, 能赋予它许多优异的特性; 在生物化学、医学、分析化 学、合成化学、材料科学等领域有着广泛的应用 ${ }^{[2]}$. 卟 啉可以作为分子器件材料 ${ }^{[3]}$ 、液晶材料、半导体材料等, 已成为材料领域的研究热点, 近年来我们课题组 ${ }^{[4,5]}$ 一 直从事于卟啉材料的研究工作. 20 世纪 80 年代末 Fox 等 ${ }^{[6]}$ 先后合成了八酯取代卟啉及其 $\mathrm{Zn}$ 配合物、八辛醚 卟啉及其配合物并研究了其液晶性, 1990 年 Kugimiya 等 ${ }^{[7]}$ 报道了对-烷氧取代型四苯基卟啉 $(n=10,12)$ 及其
Co, $\mathrm{Zn}$ 配合物 $(n=10)$ 的液晶性. 1993 年 Shimizu 等 ${ }^{[8]}$ 系 统研究了四(对烷基苯基)卟啉的液晶性. 2002 年, Velasco 小组 ${ }^{[9]}$ 报道了第一个 $\beta$ 取代型卟啉液晶衍生物. 2007 年由柳巍、师同顺等 ${ }^{[10]}$ 合成出了相变温度较低, 相 区较宽的卟啉液晶. 近年来, 人们对卟啉液晶性能做了 比较多的研究 ${ }^{[1]}$. 在前期工作中我们研究了四(对烷氧 基苯基)卟啉及其 $\mathrm{Cu}, \mathrm{Co}, \mathrm{Zn}, \mathrm{Pb}, \mathrm{Ni}, \mathrm{Al}$ 配合物的合成、 表征、液晶性能, 发现了一系列性能良好的液晶材料 ${ }^{[5]}$. 本文合成了不同链长的 meso-四(对烷氧基苯基)叶啉合 镁配合物 10 个, 用 UV-Vis, ${ }^{1} \mathrm{H} N M R, I R, M S$, 元素分析 等表征确证了该系列配合物的结构, 用差示扫描量热法

*E-mail: zhaohbhanlf@163.com

Received October 7, 2011; revised December 13, 2011; published online December 23, 2011.

Project supported by the Scientific Research Project of Xiangtan of Hunan Province (No. JZ200811) and the Natural Science Foundation of Hunan Province of China (No. 04JJ40013).

湘潭市科研课题(No. JZ200811)和湖南省自然科学基金(No. 04JJ40013)资助项目. 
(DSC) 和偏光显微镜 $(P M)$ 研究了该系列配合物的液晶 性. 合成反应式如 Eq. 1 所示.

\section{1 结果与讨论}

\section{1 配合物的液晶性能研究}

我们对所合成的系列卟啉镁配合物采用 DSC 和 PM 对其液晶性能进行了研究, 该系列配合物的相变温度、 热焓、相变区间 $(\Delta T$, DSC 数据)实验结果见表 1 , 部分配 合物的 PM 光学织构照片分别见图 1 .

结果表明在所合成的配合物中有 6 个具有液晶性, 它们有 $1 \sim 3$ 个中介相, 相变区间 $\Delta T$ 最宽为 $208.71{ }^{\circ} \mathrm{C}$,
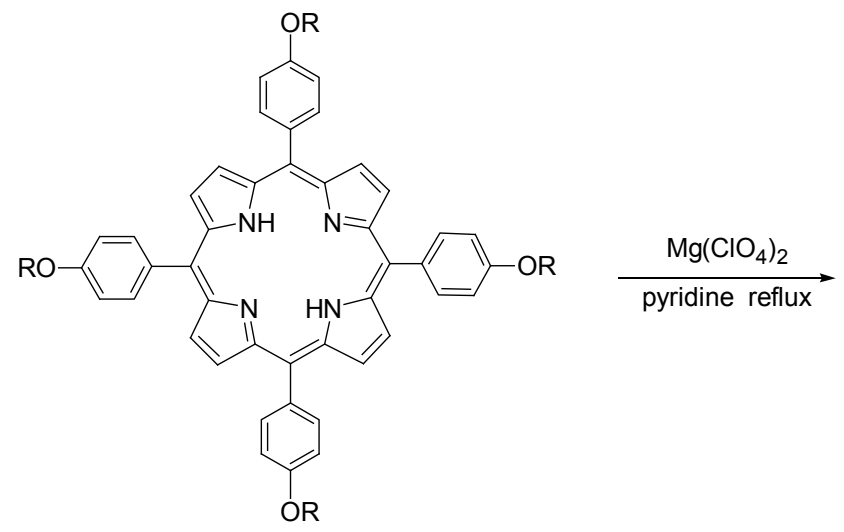

$\Delta T$ 最窄为 $19.94{ }^{\circ} \mathrm{C}$; 液晶起始相变温度最高为 166.48 ${ }^{\circ} \mathrm{C}$, 最低为 $83.97{ }^{\circ} \mathrm{C}$. 通过 $\mathrm{PM}$ 观察该系列化合物液晶 态为丝状织构(图 1).

与我们报道过的 $\mathrm{Cu}, \mathrm{Co}, \mathrm{Zn}, \mathrm{Pb}, \mathrm{Ni}$ 卟啉配合物的液 晶现象和典型的液晶相转变(互变液晶相和降温单变液 晶相 ${ }^{[5]}$ 有明显不同，本文所合成的配合物均表现为 “升 温单变液晶”. DSC 在升温时有 $1 \sim 3$ 个相转变, 但在降 温时从各向同性液体降温至 $-20{ }^{\circ} \mathrm{C}$ 时均无相转变出现, 此现象也为 PM 观察所证实, 即升温时可观察到双折射 光学织构的变化, 清亮点后再降温均不出现双折射光学 各向异性，在降温过程中视场始终是暗的. 配合物表现

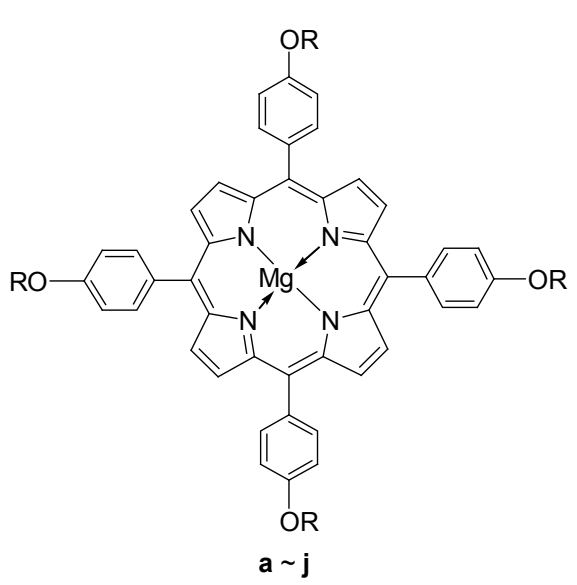

$\mathrm{R}=n-\mathrm{C}_{4} \mathrm{H}_{9}(\mathbf{a}), \mathrm{C}_{5} \mathrm{H}_{11}(\mathbf{b}), \mathrm{C}_{6} \mathrm{H}_{13}(\mathbf{c}), \mathrm{C}_{7} \mathrm{H}_{15}(\mathbf{d}), \mathrm{C}_{8} \mathrm{H}_{17}(\mathbf{e}), \mathrm{C}_{10} \mathrm{H}_{21}(\mathbf{f}), \mathrm{C}_{12} \mathrm{H}_{25}(\mathbf{g}), \mathrm{C}_{14} \mathrm{H}_{29}(\mathbf{h})$,

$\mathrm{C}_{16} \mathrm{H}_{33}(\mathbf{i}), \mathrm{C}_{18} \mathrm{H}_{37}(\mathbf{j})$

表 1 卟啉镁配合物的相变温度及热焓

Table 1 Calorimetric data for compounds $\mathbf{a} \sim \mathbf{h}$

\begin{tabular}{clc}
\hline Compd. & \multicolumn{1}{c}{$T /{ }^{\circ} \mathrm{C}\left[\Delta H /\left(\mathrm{kJ}^{\prime} \mathrm{mol}^{-1}\right)\right]$} & $\Delta T /{ }^{\circ} \mathrm{C}$ \\
\hline $\mathbf{a}$ & $\mathrm{C} 165.56(183.67), \mathrm{D} 209.05(17.31), \mathrm{I}$ & 43.39 \\
$\mathbf{b}$ & $\mathrm{C} 166.48(27.42), \mathrm{D}_{1} 183.68(13.03), \mathrm{D}_{2} 327.51(14.52), \mathrm{D}_{3}, 375.19(115.90), \mathrm{I}$ & 208.71 \\
$\mathbf{c}$ & $\mathrm{C} 138.00(17.65), \mathrm{D} 250.76(43.15), \mathrm{I}$ & 112.76 \\
$\mathbf{d}$ & $\mathrm{C} 151.89(93.37), \mathrm{D} 248.28(71.98), \mathrm{I}$ & 96.39 \\
$\mathbf{g}$ & $\mathrm{C} 83.97(20.06), \mathrm{D}_{1} 109.91(16.11), \mathrm{D}_{2} 141.12(18.22), \mathrm{I}$ & 57.15 \\
$\mathbf{h}$ & $\mathrm{C} 110.71(125.42), \mathrm{D} 130.65(12.67), \mathrm{I}$ & 19.94 \\
\hline
\end{tabular}
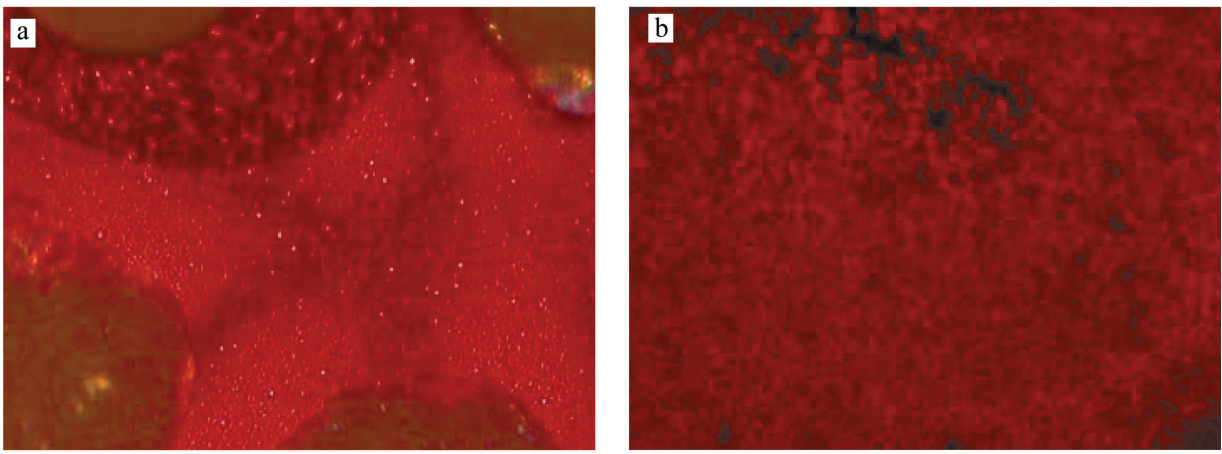

图 1 h 在 $90{ }^{\circ} \mathrm{C}$ (a)和 $125{ }^{\circ} \mathrm{C}$ (b)温度下的光学织构

Figure 1 Optical textures of $\mathbf{h}$ in the different temperatures $90{ }^{\circ} \mathrm{C}$ (a) and $125{ }^{\circ} \mathrm{C}$ (b) 
出的 “升温单变液晶”, 原因与其结构有关. 就镁卟啉 配合物而言, 因为镁原子不能嵌入卟啉环平面内, 而是 镶嵌在平面之上, 形成金字塔型的四齿配合物, 卟啉环 被扭曲，不利于分子的取向有序，其取向有序过程非常 慢, 在降温过程中这种转变的能量变化很小, 所以在 $\mathrm{DSC}$ 曲线上反映不出变化.

具有液晶性的 6 个配合物相对于配体来说表现出了 较好的液晶性能. 这是由于配位金属离子以后, 卟啉分 子形成了负电荷包围正电荷的结构, 卟啉分子存在着一 种由一个环的 $\pi$ 电子云与另一环中心的金属离子相互叠 加的倾向, 这有利于卟啉分子形成柱状排列, 从而使配 合物表现出较好的液晶性. 在这 6 个配合物中的 $\mathbf{b} \sim \mathbf{d}$, $\mathbf{h}, \mathbf{g}$ 的液晶性能随着烷氧基链的增长出现了衰减现象, 其原因是碳链的增长使脂肪族尾链的柔性增加, 在加热 熔化时烷基链较长的配合物的尾链更容易无序摆动不 利于液晶相的形成. 综上所述, 碳链数为 5 的配合物 $\mathbf{b}$ 的液晶性能最佳 $\left(\Delta T\right.$ 为 $\left.208.71{ }^{\circ} \mathrm{C}\right)$, 且有 3 个中介相.

\section{2 配合物的波谱分析}

所合成的系列镁配合物的 UV-Vis 吸收峰 $\lambda_{\text {max }}$ 在 428.2 (Soret 带), 567.0, 608.6 nm 左右, 与配体相比 Soret 带 $\left(\lambda_{\max }=423.0 \mathrm{~nm}\right.$ ) 发生了红移(约 $5 \mathrm{~nm}$ ), Q 带减少成两 个, 这说明配合物已生成. 该系列配合物的红外光谱与 配体比较, 自由碱卟啉在 $3320 \mathrm{~cm}^{-1}$ (弱)的卟啉环内 $\mathrm{N}-$ $\mathrm{H}$ 的伸缩振动吸收峰 $v_{\mathrm{N}-\mathrm{H}}$ 和在 $967 \mathrm{~cm}^{-1}$ (中强)的 $\mathrm{N}-\mathrm{H}$ 弯曲振动吸收峰 $\delta_{\mathrm{N}-\mathrm{H}}$ 消失, 取而代之是在 996 $\mathrm{cm}^{-1}$ 左右出现一个强的吸收峰(这是由于形成 $\mathrm{N}-\mathrm{Mg}$ 键而产生卟啉环骨架振动的吸收峰), 这与其它系列金 属配合物相似, 说明 $\mathrm{N}-\mathrm{H}$ 键已断裂, $\mathrm{N}-\mathrm{Mg}$ 键已生成, 且无母体残留(通过 $966 \mathrm{~cm}^{-1}$ 处有否残留吸收峰).

系列镁配合物的 ${ }^{1} \mathrm{H}$ NMR 在 $\delta-2.75$ 处 $(\delta 0 \sim$ -4 均无峰)的卟啉环内 $\mathrm{N}-\mathrm{H}$ 的吸收峰消失, 说明镁配 合物已生成, $\mathrm{N}-\mathrm{H}$ 键已断裂. 从 ${ }^{1} \mathrm{H}$ NMR 图可知, 配合 物纯净无杂质, 其吸收峰谱线变宽(成宽的单峰), 但烷 氧基上的亚甲基、甲基上的 $\mathrm{H}$ 原子种类(位置)、个数(面 积)均能很好相符. 整个对位镁配合物的 ${ }^{1} \mathrm{H}$ NMR 基本 一致，都有相同的现象和规律.

我们对该系列配合物采用激光解吸飞行时间质谱 进行质谱分析, 其分析结果为每个化合物均能在最高质 量端给出 $[\mathrm{M}]^{+}$基峰, 而其低质量端碎片峰甚少, 且丰度 很低, 不能找出其裂解规律. 该系列配合物的元素分析 结果与所设计的化合物的实验式(分子式)组成相符.

综上所述，我们所合成的配合物的 UV-Vis, ${ }^{1} \mathrm{H}$ NMR, IR, MS, 元素分析等表征结果与设计的化合物结 构相符, 其结构是可确证的.

\section{2 实验部分}

\section{1 仪器与试剂}

瑞士 Bruker Avance $400 \mathrm{MHz}$ 核磁共振仪 $\left(\mathrm{CDCl}_{3}\right.$ 为 溶剂, TMS 为内标), LDI-1700 激光解吸电离飞行时间质 谱仪, PE-2400 型元素分析仪, PE-Spectrum One 型傅立 叶变换红外光谱仪 $(\mathrm{KBr}$ 涂膜法), Lambda 25 型紫外-可 见光分光光度计 $\left(\mathrm{CH}_{2} \mathrm{Cl}_{2}\right.$ 为溶剂), $\mathrm{X}-4$ 数显显微熔点仪, DSC Q10 型差示扫描量热仪(升温和降温速率 10 ${ }^{\circ} \mathrm{C} \cdot \mathrm{min}^{-1}$ ), Leitz Laborlux12 POL 偏光显微镜(带加热台 和显微摄影装置, 升温速率 $10{ }^{\circ} \mathrm{C} \cdot \mathrm{min}^{-1}$ ).

系列 meso-四(对烷氧基苯基)卟啉(自制), 高氯酸镁 (化学纯), 二氯甲烷(分析纯), 甲醇(分析纯), 中性 $\mathrm{Al}_{2} \mathrm{O}_{3}$ (100 200 目, 层析用), 其它试剂均为市售化学纯.

\section{2 meso-四(对烷氧基苯基)卟啉的合成}

参照文献[5]合成对烷氧基苯甲醛 (4- $\mathrm{ROC}_{6} \mathrm{H}_{4} \mathrm{CHO}$ ) 10 个, 合成 meso-四 (对烷氧基苯基) 卟啉 [meso$\left.\mathrm{T}(4-\mathrm{ROP}) \mathrm{PH}_{2}\right] 10$ 个, $\mathrm{R}$ 的碳原子数分别为 $4 \sim 8,10,12$, 14, 16, 18.

\section{$2.3 m e s o$-四(对烷氧基苯基)卟啉合镁配合物的合成}

在装有空气回流冷凝管的 $50 \mathrm{~mL}$ 圆底烧瓶中, 加入 $0.02 \mathrm{mmol}$ meso-T(4-ROP)PH $\mathrm{PH}_{2}, 0.2 \mathrm{mmol}$ 高氯酸镁和 20 $\mathrm{mL}$ 吡啶, 磁力搅拌, 加热回流, 用 TLC 监测反应终点, 待原料斑点消失后(约 $8 \mathrm{~h}$ ), 停止加热. 反应完毕后减压 蒸干溶剂, 加入 $20 \mathrm{~mL}$ 水, 过滤, 用水洗涤滤饼, 得到 meso-四 (对烷氧基苯基) 卟啉镁配合物 [meso- $\mathrm{T}$ (4$\mathrm{ROP}) \mathrm{PMg}] 10$ 个, 均为紫色固体.

产物用显微熔点仪测定熔点, 用红外、紫外、核磁、 质谱、元素分析确定结构, 用 DSC 和 PM 研究其液晶性. 目标化合物的收率, 熔点, UV-Vis, ${ }^{1} \mathrm{H}$ NMR, IR, MS, 元 素分析数据如下.

\section{4 波谱数据}

meso-四(对正丁烷氧基苯基)卟啉合镁(2a): 紫色固 体, 产率 89\%. m.p. 224 225 ${ }^{\circ} \mathrm{C}$; UV-Vis $\left(\mathrm{CH}_{2} \mathrm{Cl}_{2}\right) \lambda_{\text {max }}$ : 428 (Soret), 567, $609 \mathrm{~nm} ;{ }^{1} \mathrm{H}$ NMR $\left(\mathrm{CDCl}_{3}, 400 \mathrm{MHz}\right) \delta$ : $1.12\left(\mathrm{~s}, 12 \mathrm{H}, \mathrm{CH}_{3}\right), 1.67$ (s, $\left.8 \mathrm{H}, \mathrm{CH}_{2}\right), 1.97\left(\mathrm{~s}, 8 \mathrm{H}, \mathrm{CH}_{2}\right)$, $4.28\left(\mathrm{~s}, 8 \mathrm{H}, \mathrm{OCH}_{2}\right), 7.16(\mathrm{~s}, 8 \mathrm{H}, \operatorname{Ar}-3-\mathrm{H}), 8.09(\mathrm{~s}, 8 \mathrm{H}$, Ar-2-H), 8.87 (s, 8H, $\beta$-H); IR (KBr) $v: 2929,2852\left(v_{\mathrm{CH}_{2}}\right)$, 1606, $1505\left(v_{\mathrm{C}=\mathrm{C}}\right), 1464\left(\delta_{\mathrm{CH}_{2}}\right), 1333,1281,1247\left(v_{\mathrm{Ar}-\mathrm{O}}\right)$, $1175\left(v_{\mathrm{C}-\mathrm{O}}\right), 995\left(v_{\mathrm{N}-\mathrm{Mg}}\right), 799,723 \mathrm{~cm}^{-1}$; MS (LDI) $\mathrm{m} / z$ (\%): $923.5\left(\mathrm{M}^{+}, 100\right)$. Anal. calcd for $\mathrm{MgC}_{60} \mathrm{H}_{60} \mathrm{~N}_{4} \mathrm{O}_{4}$ : C 77.87, H 6.53, N 6.05; found C 78.17, H 6.73, N 6.25.

meso-四(对正戊烷氧基苯基)卟啉合镁(2b): 紫色固 体, 产率 $86 \%$. m.p. $>300{ }^{\circ} \mathrm{C}$; UV-Vis $\left(\mathrm{CHCl}_{3}\right) \lambda_{\text {max }}: 428$ 
(Soret), 567, $608 \mathrm{~nm} ;{ }^{1} \mathrm{H}$ NMR $\left(\mathrm{CDCl}_{3}, 400 \mathrm{MHz}\right) \delta: 1.03$ $\left(\mathrm{s}, 12 \mathrm{H}, \mathrm{CH}_{3}\right), 1.17 \sim 1.60\left[\mathrm{~m}, 16 \mathrm{H},\left(\mathrm{CH}_{2}\right)_{2}\right], 1.98(\mathrm{~s}, 8 \mathrm{H}$, $\left.\mathrm{CH}_{2}\right), 4.24\left(\mathrm{~s}, 8 \mathrm{H}, \mathrm{OCH}_{2}\right), 7.16(\mathrm{~s}, 8 \mathrm{H}, \mathrm{Ar}-3-\mathrm{H}), 8.09$ (s, $8 \mathrm{H}, \mathrm{Ar}-2-\mathrm{H}), 8.87$ (s, 8H, $\beta$-H); IR (KBr) $v: 2925,2851$ $\left(v_{\mathrm{CH}_{2}}\right), 1605,1505\left(v_{\mathrm{C}=\mathrm{C}}\right), 1465\left(\delta_{\mathrm{CH}_{2}}\right), 1332,1281,1247$ $\left(v_{\mathrm{Ar}-\mathrm{O}}\right), 1174\left(v_{\mathrm{C}-\mathrm{O}}\right), 994\left(v_{\mathrm{N}-\mathrm{Mg}}\right), 797,722 \mathrm{~cm}^{-1}$; MS (LDI) $m / z \quad(\%): 980.0 \quad\left(\mathrm{M}^{+}, 100\right)$. Anal. calcd for $\mathrm{MgC}_{64} \mathrm{H}_{68} \mathrm{~N}_{4} \mathrm{O}_{4}$ : C 78.31, H 6.98, N 5.71; found C 78.61, $\mathrm{H} 6.58, \mathrm{~N} 5.73$.

meso-四(对正己烷氧基苯基)卟啉合镁(2c)：紫色固 体, 产率 88\%. m.p. $261 \sim 262{ }^{\circ} \mathrm{C}$; UV-Vis $\left(\mathrm{CHCl}_{3}\right) \lambda_{\max }$ : 428 (Soret), 567, $609 \mathrm{~nm} ;{ }^{1} \mathrm{H}$ NMR $\left(\mathrm{CDCl}_{3}, 400 \mathrm{MHz}\right) \delta$ : $0.99\left(\mathrm{t}, J=6.8 \mathrm{~Hz}, 12 \mathrm{H}, \mathrm{CH}_{3}\right), 1.26 \sim 1.62[\mathrm{~m}, 24 \mathrm{H}$, $\left.\left(\mathrm{CH}_{2}\right)_{3}\right], 2.00\left(\mathrm{~s}, 8 \mathrm{H}, \mathrm{CH}_{2}\right), 4.26\left(\mathrm{t}, J=6.4 \mathrm{~Hz}, 8 \mathrm{H}, \mathrm{OCH}_{2}\right)$, 7.22 (s, 8H, Ar-3-H), 8.09 (d, $J=8.4$ Hz, 8H, Ar-2-H), 8.97 (s, 8H, $\beta$-H); IR (KBr) v: 2925, $2855\left(v_{\mathrm{CH}_{2}}\right), 1605$, $1505\left(v_{\mathrm{C}=\mathrm{C}}\right), 1465\left(\delta_{\mathrm{CH}_{2}}\right), 1337,1281,1247\left(v_{\mathrm{Ar}-\mathrm{O}}\right), 1174$ $\left(v_{\mathrm{C}-\mathrm{O}}\right), 994\left(v_{\mathrm{N}-\mathrm{Mg}}\right), 797,722 \mathrm{~cm}^{-1}$; MS (LDI) $\mathrm{m} / z(\%)$ : $1036.3\left(\mathrm{M}^{+}\right.$, 100). Anal. calcd for $\mathrm{MgC}_{68} \mathrm{H}_{76} \mathrm{~N}_{4} \mathrm{O}_{4}: \mathrm{C}$ 78.71, H 7.38, N 5.40; found C 79.01, H 7.54, N 5.63.

meso-四(对正庚烷氧基苯基)卟啉合镁(2d): 紫色固 体, 产率 85\%. m.p. 252 254 ${ }^{\circ} \mathrm{C}$; UV-Vis $\left(\mathrm{CHCl}_{3}\right) \lambda_{\text {max }}$ : 428 (Soret), 567, $609 \mathrm{~nm} ;{ }^{1} \mathrm{H}$ NMR $\left(\mathrm{CDCl}_{3}, 400 \mathrm{MHz}\right) \delta$ : $0.95\left(\mathrm{~s}, 12 \mathrm{H}, \mathrm{CH}_{3}\right), 1.38 \sim 1.61\left[\mathrm{~m}, 32 \mathrm{H},\left(\mathrm{CH}_{2}\right)_{4}\right], 1.95(\mathrm{~s}$, $\left.8 \mathrm{H}, \mathrm{CH}_{2}\right), 4.22\left(\mathrm{~s}, 8 \mathrm{H}, \mathrm{OCH}_{2}\right), 7.32$ (s, 8H, Ar-3-H), 8.08 (s, 8H, Ar-2-H), 8.88 (s, 8H, $\beta$-H); IR (KBr) v: 2926, 2856 $\left(v_{\mathrm{CH}_{2}}\right), 1607,1505\left(v_{\mathrm{C}=\mathrm{C}}\right), 1464\left(\delta_{\mathrm{CH}_{2}}\right), 1331,1280,1245$ $\left(v_{\mathrm{Ar}-\mathrm{O}}\right), 1174\left(v_{\mathrm{C}-\mathrm{O}}\right), 995\left(v_{\mathrm{N}-\mathrm{Mg}}\right), 797,723 \mathrm{~cm}^{-1}$; MS (LDI) $m / z$ (\%): $1091.2\left(\mathrm{M}^{+}, 100\right)$. Anal. calcd for $\mathrm{MgC}_{72} \mathrm{H}_{84} \mathrm{~N}_{4} \mathrm{O}_{4}$ : C 79.06, H 7.74, N 5.12; found C 79.36, H 7.46, N 5.02 .

meso-四(对正辛烷氧基苯基)卟啉合镁 $[2 \mathrm{e}]$ : 紫色固 体, 产率 86\%. m.p. $210 \sim 212{ }^{\circ} \mathrm{C}$; UV-Vis $\left(\mathrm{CHCl}_{3}\right) \lambda_{\text {max }}$ : 428 (Soret), 567, $609 \mathrm{~nm} ;{ }^{1} \mathrm{H}$ NMR $\left(\mathrm{CDCl}_{3}, 400 \mathrm{MHz}\right) \delta$ : $0.89\left(\mathrm{~s}, 12 \mathrm{H}, \mathrm{CH}_{3}\right), 1.29 \sim 1.62\left[\mathrm{~m}, 40 \mathrm{H},\left(\mathrm{CH}_{2}\right)_{5}\right], 1.97(\mathrm{~s}$, $\left.8 \mathrm{H}, \mathrm{CH}_{2}\right), 4.26\left(\mathrm{~s}, 8 \mathrm{H}, \mathrm{OCH}_{2}\right), 7.22$ (s, 8H, Ar-3-H), 8.08 (s, 8H, Ar-2-H), 8.98 (s, 8H, $\beta$-H); IR (KBr) v: 2924, 2855 $\left(v_{\mathrm{CH}_{2}}\right), 1607,1506\left(v_{\mathrm{C}=\mathrm{C}}\right), 1463\left(\delta_{\mathrm{CH}_{2}}\right), 1332,1281,1247$ $\left(v_{\mathrm{Ar}-\mathrm{O}}\right), 1175\left(v_{\mathrm{C}-\mathrm{O}}\right), 996\left(v_{\mathrm{N}-\mathrm{Mg}}\right), 797,724 \mathrm{~cm}^{-1} ; \mathrm{MS}$ (LDI) $m / z(\%): 1147.5\left(\mathrm{M}^{+}, 100\right)$. Anal. calcd for $\mathrm{MgC}_{76^{-}}$ $\mathrm{H}_{92} \mathrm{~N}_{4} \mathrm{O}_{4}$ : C 79.38, $\mathrm{H}$ 8.06, N 4.87; found C 79.66, H 8.35, $\mathrm{N} 4.80$.

meso-四(对正癸烷氧基苯基)卟啉合镁(2f)：紫色固 体, 产率 88\%. m.p. $185 \sim 186{ }^{\circ} \mathrm{C}$; UV-Vis $\left(\mathrm{CHCl}_{3}\right) \lambda_{\max }$ : 428 (Soret), 567, $608 \mathrm{~nm} ;{ }^{1} \mathrm{H}$ NMR $\left(\mathrm{CDCl}_{3}, 400 \mathrm{MHz}\right) \delta$ : $0.91\left(\mathrm{~s}, 12 \mathrm{H}, \mathrm{CH}_{3}\right), 1.19 \sim 1.62\left[\mathrm{~m}, 56 \mathrm{H},\left(\mathrm{CH}_{2}\right)_{7}\right], 1.96(\mathrm{~s}$, $\left.8 \mathrm{H}, \mathrm{CH}_{2}\right), 4.24\left(\mathrm{~s}, 8 \mathrm{H}, \mathrm{OCH}_{2}\right), 7.23$ (s, 8H, Ar-3-H), 8.11 (s, 8H, Ar-2-H), 8.89 (s, 8H, $\beta$-H); IR (KBr) v: 2922, 2852 $\left(v_{\mathrm{CH}_{2}}\right), 1605,1505\left(v_{\mathrm{C}=\mathrm{C}}\right), 1466\left(\delta_{\mathrm{CH}_{2}}\right), 1332,1280,1247$ $\left(v_{\mathrm{Ar}-\mathrm{O}}\right), 1175\left(v_{\mathrm{C}-\mathrm{O}}\right), 995\left(v_{\mathrm{N}-\mathrm{Mg}}\right), 797,722 \mathrm{~cm}^{-1} ; \mathrm{MS}$ (LDI) $m / z \quad(\%)$ : $1259.1 \quad\left(\mathrm{M}^{+}, 100\right)$. Anal. calcd for $\mathrm{MgC}_{84} \mathrm{H}_{108} \mathrm{~N}_{4} \mathrm{O}_{4}$ : C 79.94, $\mathrm{H}$ 8.63, $\mathrm{N} 4.44$; found $\mathrm{C} 79.84$, H 8.94, N 4.38.

meso-四(对正十二烷氧基苯基)卟啉合镁 $(\mathbf{2 g})$ ：紫色 固体, 产率 $89 \%$. m.p. $165 \sim 166{ }^{\circ} \mathrm{C}$; UV-Vis $\left(\mathrm{CHCl}_{3}\right)$ $\lambda_{\text {max }}$ : 428 (Soret), 567, $609 \mathrm{~nm} ;{ }^{1} \mathrm{H}$ NMR $\left(\mathrm{CDCl}_{3}, 400\right.$ $\mathrm{MHz}) \delta: 0.88\left(\mathrm{~s}, 12 \mathrm{H}, \mathrm{CH}_{3}\right), 1.03 \sim 1.62\left[\mathrm{~m}, 72 \mathrm{H},\left(\mathrm{CH}_{2}\right)_{9}\right]$, $1.96\left(\mathrm{~s}, 8 \mathrm{H}, \mathrm{CH}_{2}\right), 4.24\left(\mathrm{~s}, 8 \mathrm{H}, \mathrm{OCH}_{2}\right), 7.21(\mathrm{~s}, 8 \mathrm{H}$, Ar-3-H), 8.09 (s, 8H, Ar-2-H), 8.88 (s, 8H, $\beta$-H); IR (KBr) $v: 2923,2853\left(v_{\mathrm{CH}_{2}}\right), 1605,1505\left(v_{\mathrm{C}=\mathrm{C}}\right), 1464\left(\delta_{\mathrm{CH}_{2}}\right), 1332$, 1280, $1247\left(v_{\mathrm{Ar}-\mathrm{O}}\right), 1175\left(v_{\mathrm{C}-\mathrm{O}}\right), 996\left(v_{\mathrm{N}-\mathrm{Mg}}\right), 797,721$ $\mathrm{cm}^{-1}$; MS (LDI) $\mathrm{m} / z(\%): 1370.2\left(\mathrm{M}^{+}, 100\right)$. Anal. calcd for $\mathrm{MgC}_{92} \mathrm{H}_{124} \mathrm{~N}_{4} \mathrm{O}_{4}$ : C 80.40, $\mathrm{H}$ 9.09, $\mathrm{N}$ 4.08; found $\mathrm{C}$ $80.25, \mathrm{H} 9.23$, N 4.05 .

meso-四(对正十四烷氧基苯基)卟啉合镁(2h)：紫色 固体, 产率 87\%. m.p. $141 \sim 142{ }^{\circ} \mathrm{C}$; UV-Vis $\left(\mathrm{CHCl}_{3}\right)$ $\lambda_{\max }: 428$ (Soret), 567, $609 \mathrm{~nm} ;{ }^{1} \mathrm{H} \mathrm{NMR}\left(\mathrm{CDCl}_{3}, 400\right.$ $\mathrm{MHz}) \delta: 0.88\left(\mathrm{~s}, 12 \mathrm{H}, \mathrm{CH}_{3}\right), 1.28 \sim 1.88[\mathrm{~m}, 88 \mathrm{H}$, $\left.\left(\mathrm{CH}_{2}\right)_{11}\right], 1.93\left(\mathrm{t}, J=7.2 \mathrm{~Hz}, 8 \mathrm{H}, \mathrm{CH}_{2}\right), 4.23(\mathrm{t}, J=6.0 \mathrm{~Hz}$, $\left.8 \mathrm{H}, \mathrm{OCH}_{2}\right), 7.23$ (s, 8H, Ar-3-H), 8.09 (d, $J=8.4 \mathrm{~Hz}, 8 \mathrm{H}$, Ar-2-H), 8.86 (s, 8H, $\beta$-H); IR (KBr) v: 2923, $2854\left(v_{\mathrm{CH}_{2}}\right)$, $1607,1499\left(v_{\mathrm{C}=\mathrm{C}}\right), 1469\left(\delta_{\mathrm{CH}_{2}}\right), 1333,1281,1248\left(v_{\mathrm{Ar}-\mathrm{O}}\right)$, $1175\left(v_{\mathrm{C}-\mathrm{O}}\right), 995\left(v_{\mathrm{N}-\mathrm{Mg}}\right), 800,722 \mathrm{~cm}^{-1}$; MS (LDI) $\mathrm{m} / z$ (\%): $1482.6\left(\mathrm{M}^{+}, 100\right)$. Anal. calcd for $\mathrm{MgC}_{100} \mathrm{H}_{140} \mathrm{~N}_{4} \mathrm{O}_{4}$ : C 80.80, H 9.49, N 3.77; found C 80.92, H 9.61, N 3.87.

meso-四(对正十六烷氧基苯基)卟啉合镁(2i): 紫色 固体, 产率 83\%. m.p. $131 \sim 133{ }^{\circ} \mathrm{C}$, UV-Vis $\left(\mathrm{CHCl}_{3}\right)$ $\lambda_{\max }: 428$ (Soret), 567, $608 \mathrm{~nm} ;{ }^{1} \mathrm{H}$ NMR $\left(\mathrm{CDCl}_{3}, 400\right.$ $\mathrm{MHz}) \delta: 0.87\left(\mathrm{~s}, 12 \mathrm{H}, \mathrm{CH}_{3}\right), 1.26 \sim 1.59[\mathrm{~m}, 104 \mathrm{H}$, $\left.\left(\mathrm{CH}_{2}\right)_{13}\right], 1.94\left(\mathrm{~s}, 8 \mathrm{H}, \mathrm{CH}_{2}\right), 4.23\left(\mathrm{~s}, 8 \mathrm{H}, \mathrm{OCH}_{2}\right), 7.15$ (s, $8 \mathrm{H}, \mathrm{Ar}-3-\mathrm{H}), 8.09$ (s, 8H, Ar-2-H), 8.88 (s, $8 \mathrm{H}, \beta-\mathrm{H})$; IR $(\mathrm{KBr}) v: 2922,2852\left(v_{\mathrm{CH}_{2}}\right), 1602,1507\left(v_{\mathrm{C}=\mathrm{C}}\right), 1467\left(\delta_{\mathrm{CH}_{2}}\right)$, 1332, 1280, $1245\left(v_{\mathrm{Ar}-\mathrm{O}}\right), 1174\left(v_{\mathrm{C}-\mathrm{O}}\right), 996\left(v_{\mathrm{N}-\mathrm{Mg}}\right), 797$, $721 \mathrm{~cm}^{-1}$; MS (LDI) m/z (\%): $1597.0\left(\mathrm{M}^{+}, 100\right)$. Anal. calcd for $\mathrm{MgC}_{108} \mathrm{H}_{156} \mathrm{~N}_{4} \mathrm{O}_{4}$ : C 81.14, H 9.84, N 3.50; found C 81.24, H 9.85, N 3.55.

meso-四(对正十八烷氧基苯基)卟啉合镁 $(2 \mathbf{j})$ : 紫色 固体，产率 84\%. m.p. 85 89 ${ }^{\circ} \mathrm{C}$; UV-Vis $\left(\mathrm{CHCl}_{3}\right) \lambda_{\max }$ : 428 (Soret), 566, $608 \mathrm{~nm} ;{ }^{1} \mathrm{H}$ NMR $\left(\mathrm{CDCl}_{3}, 400 \mathrm{MHz}\right) \delta$ : $0.87\left(\mathrm{~s}, 12 \mathrm{H}, \mathrm{CH}_{3}\right), 1.16 \sim 1.95\left[\mathrm{~m}, 120 \mathrm{H},\left(\mathrm{CH}_{2}\right)_{15}\right], 2.12$ 
(s, 8H, $\left.\mathrm{CH}_{2}\right), 4.25\left(\mathrm{~s}, 8 \mathrm{H}, \mathrm{OCH}_{2}\right), 7.21(\mathrm{~s}, 8 \mathrm{H}, \mathrm{Ar}-3-\mathrm{H})$, 8.09 (s, 8H, Ar-2-H), 8.98 (s, 8H, $\beta$-H); IR (KBr) v: 2925, $2852\left(v_{\mathrm{CH}_{2}}\right), 1601,1506\left(v_{\mathrm{C}=\mathrm{C}}\right), 1468\left(\delta_{\mathrm{CH}_{2}}\right), 1332,1281$, $1247\left(v_{\mathrm{Ar}-\mathrm{O}}\right), 1174\left(v_{\mathrm{C}-\mathrm{O}}\right), 995\left(v_{\mathrm{N}-\mathrm{Mg}}\right), 799,720 \mathrm{~cm}^{-1}$; MS (LDI) $m / z \quad(\%): 1710.7\left(\mathrm{M}^{+}, 100\right)$. Anal. calcd for $\mathrm{MgC}_{116} \mathrm{H}_{172} \mathrm{~N}_{4} \mathrm{O}_{4}$ : C 81.43, $\mathrm{H}$ 10.13, N 3.27; found $\mathrm{C}$ 81.56, H 10.40, N 3.29 .

\section{References}

[1] Zhao, S.-F.; Chen, N.-Y.; Guo, X.; Wang, M.-H.; Li, Z.-Y. Chin. J. Org. Chem. 2010, 30(12), 1898 (in Chinese). (赵胜芳, 陈年友, 郭金金, 王孟华, 李早英, 有机化学, 2010, 30(12), 1898.)

[2] Wang. Z.-F.; Deng, W.-L. Prog. Chem. 2007, 19(4), 520 (in Chinese). (王周锋, 邓文礼, 化学进展, 2007, 19(4), 520.)

[3] Jurow, M.; Schuckman, A. E.; Batteas, J. D.; Drain, C. M. Coord. Chem. Rev. 2010, 254, 2297.

[4] (a) Wang, H.-K.; Peng, S.-M.; Zhao, H.-B.; Xu, L.-L.; Zhang, H.; Lu, X.-J. Acta Chim. Sinica 2009, 67(14), 1643 (in Chinese). (王红科, 彭圣明, 赵鸿斌, 徐兰兰, 张辉, 陆旭甲, 化学学报, 2009, 67(14), 1643.)

(b) Zhang, H.; Zhao, H.-B.; Lu, X.-J.; Wang, W.-L.; Chang, H.; Wang, Y.-F. Acta Chim. Sinica 2011, 69(3), 316 (in Chinese). (张辉, 赵鸿斌, 陆旭甲, 王武林, 常慧, 王玉芳, 化学学报, 2011, 69(3), 316.)

[5] (a) Zhao, H.-B.; Cai, J.; Lin, Y.-B.; Yang, N.-F.; Wang, X.-Y. Acta Chim. Sinica 2000, 58(5), 543 (in Chinese).

(赵鸿斌, 蔡剑, 林原斌, 阳年发, 王霞瑜, 化学学报, 2000,
58(5), 543.)

(b) Liu, J.-P.; Zhao, H.-B.; Xu, Y.-J.; Cao, H.; Chen, K. Chin. J. Org. Chem. 2007, 27(6), 778 (in Chinese).

(刘捷频, 赵鸿斌, 徐勇军, 曹华, 陈科, 有机化学, 2007, 27(6), 778.)

(c) Zhao, H.-B.; Luo, H.-A.; He, J.-P.; Peng, S.-M.; Xie, Q.-Y. Acta Chim. Sinica 2006, 64(4), 331 (in Chinese).

(赵鸿斌, 罗和安, 贺江平, 彭圣明, 谢琼玉, 化学学报, 2006, 64(4), 331.)

(d) He, J.-P.; Zhao, H.-B.; Chang, H.; Peng, S.-M.; Xie, Q.-Y.; Liu, J.-P. Chin. J. Org. Chem. 2006, 26(12), 1663 (in Chinese).

(贺江平, 赵鸿斌, 常慧, 彭圣明, 谢琼玉, 刘捷频, 有机化学, 2006, 26(12), 1663.)

(e) Xu, Y.-J.; Gu, Z.; Zhao, H.-B.; Wang, L.-S.; Ning, M.-X.; Qiu, S.-J. Chem. J. Chin. Univ. 2008, 29(10), 1930 (in Chinese).

(徐勇军, 顾峥, 赵鸿斌, 王励申, 宁满侠, 仇诗军, 高等学校化 学学报, 2008, 29(10), 1930.)

[6] (a) Gregg, B. A.; Fox, A. J. J. Chem. Soc., Chem. Commun. 1987, 15,1134 .

(b) Gregg, B. A.; Fox, M. A.; Bard, A. J. J. Am. Chem. Soc. 1989, $111,3024$.

[7] Kugimiya, S.; Takemura, M. Tetrahedron Lett. 1990, 31(22), 3157.

[8] Shimizu, Y.; Miya, M.; Nagata, A.; Ohta, K.; Yamamoto, I.; Kusabayashi, S. Liq. Cryst. 1993, 14(3), 795.

[9] Castella, M.; López-Calahorra, F.; Velasco, D.; Finkelmann, H. Chem. Commun. 2002, 20, 2348.

[10] Liu, W.; Shi, T.-S. Sci. China, Ser. B: Chem. 2007, 50(4), 488.

[11] (a) Wang, Q. M.; Bruce, D. W. Angew. Chem., Int. Ed. Engl. 1997, 36,150 .

(b) Ying, X.; Shi, J. Liq. Cryst. 2000, 27, 211.

(c) Zhao, Z.-X.; Lin, G.-F. Liq. Cryst. 2002, 29(10), 1335.

(d) Qi, M.-H.; Lin, G.-F. J. Phys. Chem. B 2003, 107(31), 7640.

(Qin, X.) 